\title{
The Method of Iterative Formation of Selective Reference Images
}

\author{
NataliiaYeromina $^{1}$, Valerii Samoilenko ${ }^{2}$, Dmytro Chukanivskyi $^{3}$, Olena Zadkova ${ }^{4}$, Olesia Brodova $^{5}$, \\ Olha Levchenko ${ }^{6}$ \\ ${ }^{1}$ Department of Electronic Computers, Kharkiv National University of Radio Electronics, Ukraine, Kharkiv, \\ nataliia.yeromina@nure.ua \\ ${ }^{2,3}$ National Academy of Ukrainian National Guards, Ukraine, samoylenko.valeriy1007@gmail.com \\ ${ }^{4,5}$ Flight Academy of the National Aviation University, Ukraine, elenazadkova71@gmail.com \\ ${ }^{6}$ State University of Infrastructure and Technologies, Ukraine,olevchenko76@ gmail.com
}

\begin{abstract}
The results of study of the method of iterative formation of selective reference images for correlation-extreme navigation systems (CENS) of aircrafts by the method of "sliding window" are presented using image correlation analysis based on brightness. The method is applicable in a CENS with external sensors forming images of the reference area in the visible optical, infrared and radar range. The results of studies of the formation and use of binary images under conditions of limitations for time and volume of calculations during formation of selective RI are given.
\end{abstract}

Key words: current image, reference area, selective image, object brightness, correlation analysis, navigation systems, "sliding window".

\section{INTRODUCTION}

Navigation of aircraft, which uses the correlation-extreme systems (CENS), depends on multiple factors, both external and internal. However, the issue of reference image (RI) formation on the referenced areas remains the most important.The type of surface and the objects located on it, which differ according to informative characteristics, such as brightness, contrast, linear dimensions, are essential.It is often necessary to bind with surfaces with a small number of objects, besides having small vertical dimensions.All these factors can have a significant impact on the accuracy of aircraft positioning, necessitating research and the search for a rational way to generate RI. In addition, these factors should be taken into considerationduring the development of databases on the visualization surface, taking into account the construction features of the external information sensors used in CENS [1, 2, 3].

\subsection{Problem analysis}

In the articles $[4,5,6,7,8,9]$ ways of forming optimum RI are proposed also taking into account algorithms of comparison of current and reference images. However, not enough attention has been paid to the specific methods and techniques of RI formation, modelling and evaluation of the performance of the resulting RI.In addition, the possibilities of producing depleted images in order to reduce the time and amount of computation have not been considered.

The object of the article is to study the practical ways of forming RI and to test them using real images of the sighting surfaces.

\section{MAIN MATERIAL}

2.1 The formation of reference images based on the brightness characteristics of reference area

It is proposed to form reference images in conditions of a limited number of objects on the surface of the reference area on the basis of the results of a correlation comparison of the original image (OI) of the reference area with a set of selective images.Furthermore, in order to identify and isolate objects (fragments of the image) with a dominant brightness, a correlation is proposedbased on the brightness parameter measured by the primary processing sensor of the information extraction system, according to which selective images of the corresponding background are formed.Such objects may, for example, be a dirt road against a field background, a river bed.In order to perform the correlation analysis of the image, it is advisable to carry out the "sliding window" method.The essence of the "sliding window" method is the general analysis of image pixels that are "covered" by some two-dimensional, usually square, area of finite size.All image pixels that enter the comparison sector are processed according to a certain rule. The result of the processing is the brightness (color intensity) of the pixel of the original image or the element of the two-dimensional matrix of the transformation results, which corresponds to the center of the window. The window is then shifted by one pixel and the processing is repeated.The process is completed when the window is shifted to all possible values within the image.If fragments of the input image itself are used alternately to perform the luminance correlation analysis of the image as a "sliding window", the result of this treatment will be a set of joint correlated function(JCF) offragments of images and whole images.Therefore, such a correlation analysis will make it possible to determine the contribution of image fragments to the autocorrelation function (ACF) of the whole image. This is necessary to produce a selective RI that 
includes only those fragments of the image that are important for maintaining the correlation between the original image and the formed RI.Thus, in order to perform the correlation analysis of the image according to the brightness as a "sliding window", we shall alternate the fragments of the original image of the size $\left(\mathrm{M}_{\mathrm{W}}, \mathrm{N}_{\mathrm{W}}\right)$. Each next fragment of the image ("window") will be offset from the previous one by one pixel horizontally (vertically). The image, correlated based on the brightness, is formed according to the expression:

$$
\mathrm{K}_{\mathrm{CCF}}(\mathrm{i}, \mathrm{j})_{\mathrm{mn}}=\sum_{\mathrm{i}_{\mathrm{i}=0}}^{\mathrm{M}-1} \sum_{\mathrm{j}=0}^{\mathrm{N}-1}\left[\mathrm{~S}_{\mathrm{OI}}(\mathrm{i}, \mathrm{j}) \mathrm{S}_{\mathrm{W}}(\mathrm{i}+\mathrm{m}, \mathrm{j}+\mathrm{n})\right],(1)
$$

where $\mathrm{K}_{\mathrm{CCF}}$ is a discrete two-dimensional cross-correlation function;

$\mathrm{M}, \mathrm{N}$ are the dimensions of $\mathrm{OI}$ in pixels;

$\mathrm{M}_{\mathrm{W}}, \mathrm{N}_{\mathrm{W}^{-}}$the dimensions of the sliding window (SW) in pixels (for calculation, the $\mathrm{SW}$ is supplemented by zeros up to the size of $\mathrm{OI}$;

$\mathrm{m}=\left[0, \ldots, \mathrm{M}-\mathrm{M}_{\mathrm{W}}\right], \mathrm{n}=\left[0, \ldots, \mathrm{N}-\mathrm{N}_{\mathrm{W}}\right]-$ displacement (shift) of SW relative to OI by the slim axes in pixels;

$S_{\mathrm{OI}}(i, j), S_{W}(i, j)-$ the brightness (intensity of grey) of the input image and its fragment (SW) at the coordinates $(i, j)$.

Figure 1 illustrates the selection process of SW and its "sliding" by OI to calculate $\mathrm{K}_{\mathrm{CCF}}(\mathrm{i}, \mathrm{j})_{\mathrm{mn}}$. The images are in the form of gray-level arrays.

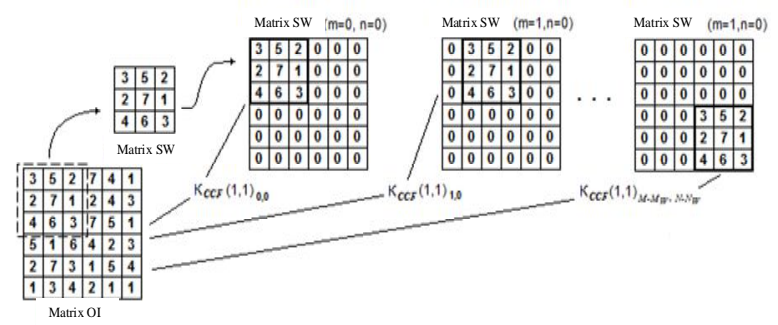

Figure 1: Illustration of the $\mathrm{K}_{\mathrm{CCF}}(\mathrm{i}, \mathrm{j})_{\mathrm{mn}}$ calculation process (OI matrix - $6 \times 6$ pixels, $S W-3 \times 3$ pixels)

The resulting functions of $\mathrm{K}_{\mathrm{CCF}}(\mathrm{i}, \mathrm{j})_{\mathrm{mn}}$ form a field of correlation analysis(FCA) by brightness (FCAB), which is a matrix $\mathrm{M}_{\mathrm{FCAB}}^{\prime}$ of size $\left(\mathrm{M}-\mathrm{M}_{\mathrm{W}}, \mathrm{N}-\mathrm{N}_{\mathrm{W}}\right)$, the elements of which are the values $\mathrm{M}_{\mathrm{FCAB}}^{\prime}(\mathrm{i}, \mathrm{j})=\max \left[\mathrm{K}_{\mathrm{CCF}}(\mathrm{i}, \mathrm{j})_{\mathrm{mn}}\right]$. In order to avoid the effect of absolute brightness values on the resulting FCAB field, it is advisable to carry out rationing of the FCA:

$$
\mathrm{M}_{\mathrm{FCAB}}=\frac{\mathrm{M}_{\mathrm{FCAB}}^{\prime}}{\max \left[\mathrm{M}_{\mathrm{FCAB}}^{\prime}\right]}
$$

If the $\mathrm{OI}$ is color one, the color image must be reduced to grayscale ("grey wedge") in order to obtain its gray-level array:

$\mathrm{S}_{\mathrm{OI}}(\mathrm{i}, \mathrm{j})^{*}=0,2989 \cdot \mathrm{S}_{\mathrm{R}}(\mathrm{i}, \mathrm{j})+0.5870 \cdot \mathrm{S}_{\mathrm{G}}(\mathrm{i}, \mathrm{j})+0.1140 \cdot \mathrm{S}_{\mathrm{B}}(\mathrm{i}, \mathrm{j})$, where $\mathrm{S}_{\mathrm{OI}}(\mathrm{i}, \mathrm{j})^{*}-$ the brightness of the pixel image in the «grey wedge»;

$S_{R}(i, j), S_{G}(i, j), S_{B}(i, j)$-intensity of red, green and blue components of color respectively of( $i, j$ )-th OI pixel (pixel ini-th column of $\mathrm{j}$-th line of $\mathrm{OI}(\mathrm{i} \in 0 . . \mathrm{M}-1, \mathrm{j} \in 0 \ldots \mathrm{N}-1$, at image size $\mathrm{M} \times \mathrm{N}$ pixels).
Figure 2 presents the result of the conversion of the test image to grayscale and the results of the calculation of the FCAB for the test image with SW $2 \times 2$ pixels. As it can be seen from the results of the FCAB calculation, the correlation analysis of the image according to the brightness allows to "illuminate" bright areas of the image.Furthermore, the brighter the fragments of the image, the more intense the "illumination" of these areas will be.

To check whether "highlighted" areas of the image actually make a significant contribution to the auto-correlation function of the image, it is necessary to select these areas on the image (get a segmented image) and determine the $\mathrm{ACF}$ of the OI and the JCF of the segmented image and compare them with each other.

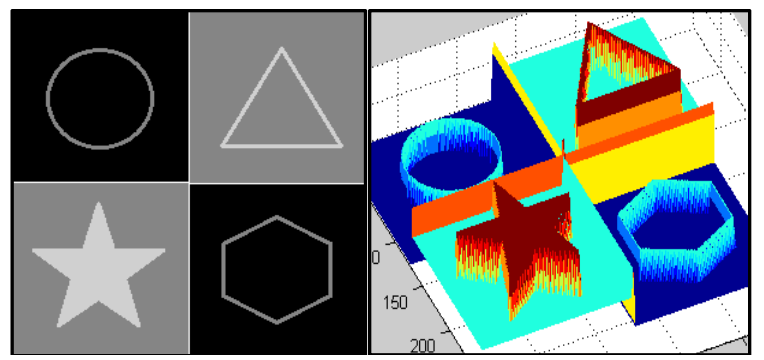

Figure 2: Test image in the "grey wedge" (left) and the result of calculating the FCAB for it, shown in the three-dimensional projection (right)

To form a selective image we will use the resulting normalized FCAB .To do this, we transform the OI in such a way as to leave only those fragments of the OI for which the level of the normalizedFCAB was greater than some level, for example 0.5 .

The result for the test image is the selective image shown in Figure 3.Geometric figures of OI, which have the greatest brightness, are shown in the selected image.On Figure 4 is given the ACF of the test image and the JCF of the test image and the selective image obtained therefrom (using the FCAB).As can be seen in figure 2, the JCF is almost entirely in compliance with the ACF.However, the JCF shows a slight narrowing of the main lobe and a marked decline in the level of the side lobes. . If this is considered a systemic phenomenon, the use of selective images as RI may have certain advantages, as it may provide better characteristics of the critical function to be calculated on board the aircraft.

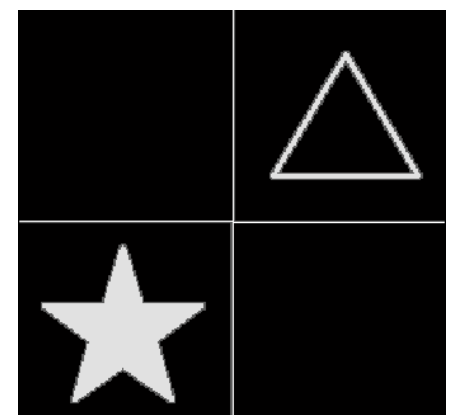

Figure 3: The selective image obtained from the test OI by crosssection of theFCAB at 0.5 . 


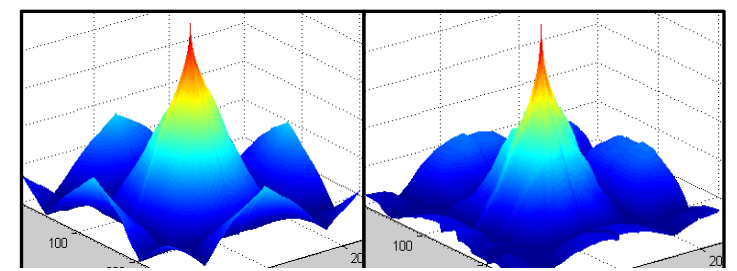

Figure 4: ACF of the test image (left) and the JCF of the test and selective images (right).

Figure 5 shows the results of the study for the real image of the surface in the optical range given in Figure 6.

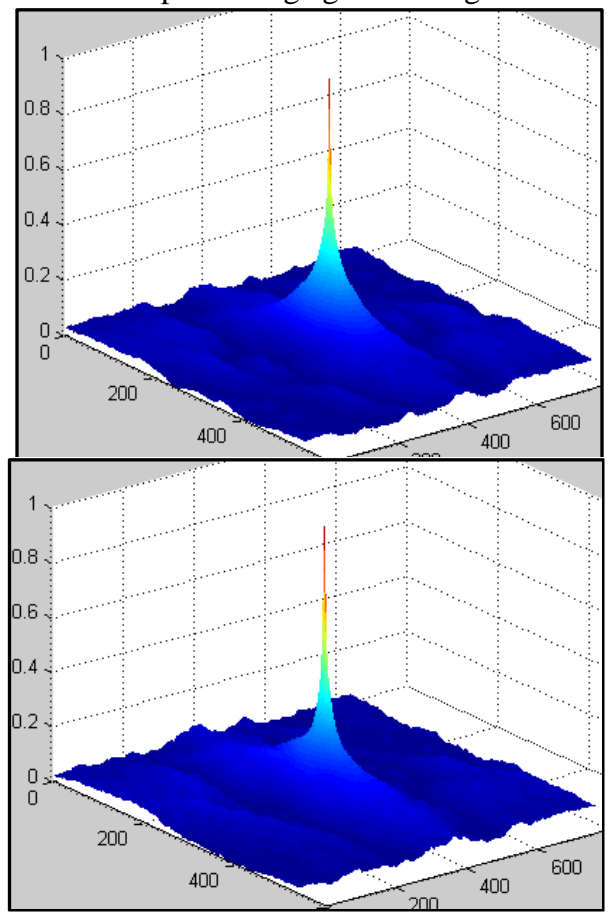

Figure 5: the ACF of OI and the JCF of original and selective images

Figure 5 - Results of selective imaging based on satellite sounder and correlation function calculations.
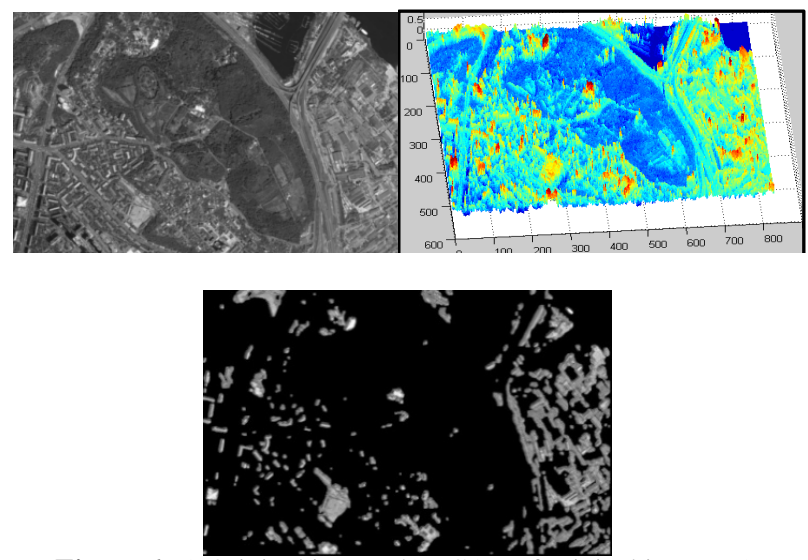

Figure 6: a) Original image; b) FCAB of original image; c) Selective image

Figure 7, 8 shows the selective image and the JCF for the same image for the 0.7 cross-section of the FCAB.

The imageis predefined to gray gradations. A FCAB crosssection level of 0.5 has been applied to obtain a selective image. As can be seen in the Figure 8, the JCF of the original and selective images visually corresponds to the $\mathrm{ACF}$ of the OI.There is a slight narrowing of the main lobe of the correlation function, which indicates a faster "breakup" of the correlation between the images while increasing the spatial shift between them.

As it can be seen in Figure 8, the results of the calculation of the JCF differ from the previous case, which shows the possibility and usefulness of selecting some rational level of the crossing of the FCAB, at which a selective image with a certain number of informative features will be obtained, on the one hand, and characterized by the preservation of a correlation between the original and selective image, on the other hand.

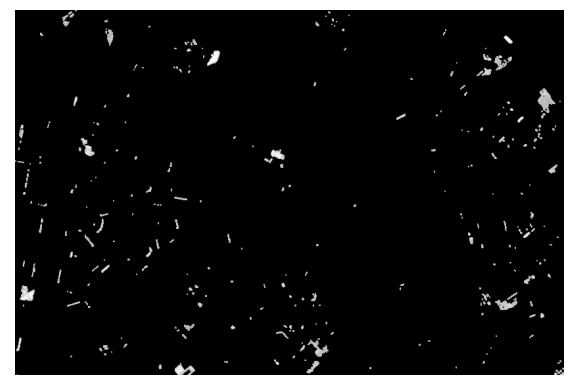

Figure 7: Selective image for FCAB section at 0.7

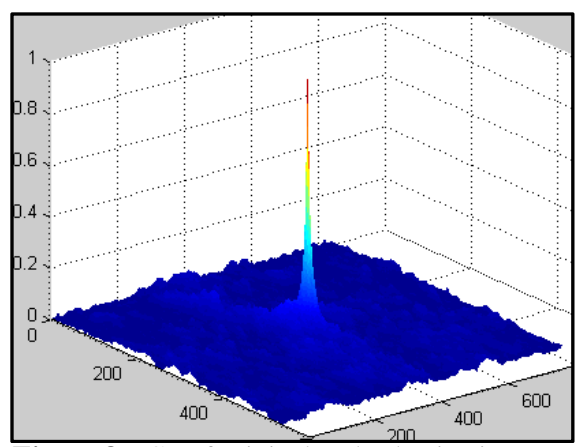

Figure 8: JCF of original and selective images

Thus, selective images obtained by highlighting the brightest areas of OI maintain a correlation with the original image.Since the FCAB cross-section level can be different, it is evident that a necessary part of the reference image generation methodis the certain quality control of the obtained selective images by constructing and comparing the ACF of the original image and the JCF of the original and selective images.

Since the visual analysis (comparison) of the ACF and the JCF of the original and selective images is subjective, it is appropriate to use a quantitative measure to quantify the $\mathrm{ACF}$ (JCF) produced, which will be used as an indicator of selective image quality.

A selective image that will have the best value of such an indicator is RI.. This generalized indicator can be the degree of dominance of the maximum of the main lobe of the correlation function above the level of its "background", which is calculated as the ratio of the main lobe level to the average level of the correlation function.Objectively, as a result of the calculations, for all the examples noted, the indicator assumed different values, namely:

For the ACF of the test image - 7;

For the JCF of the test and selective image obtained -9; 
For ACF of the real image -26;

For the JCF of a real image with a FCAB cross-section 0.5 - 27;

For the JCF of a real image with a FCAB cross-section 0.7 $-48$.

These values are distinct (indicating the sensitivity of the indicator) and fully reflect the visual estimates of the built functions.(This measure of "quality" of the correlation functions is hereinafter referred to as $\mathrm{L}_{\mathrm{Q}}$ ).

A model experiment using the developed CENS simulation model was carried out in order to confirm the possibility of forming RI using the proposed method.

The experiment used different images of the same terrain (from different satellites and obtained during different seasons) to form RI using the proposed method and to simulate the process of obtaining current images from external sensors.

The results of the simulation are shown in figure 9.

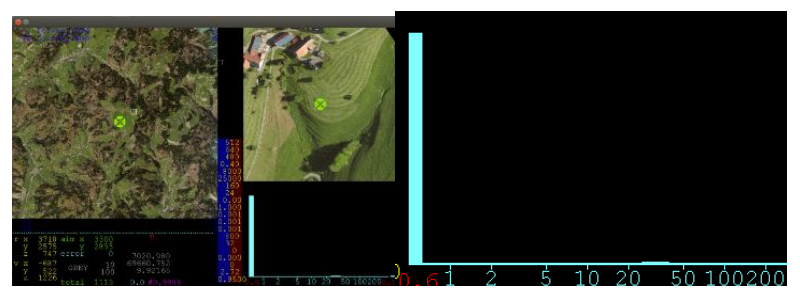

Figure 9: Modelling results using the CENS simulation model

The number of iterations of simulations was more than 1,000.The resultant value of the circular probability deviation of an aircraft equipped with CENS from the given location point was $0.6 \mathrm{~m}$, which fully meets the modern requirements for the solution of most aircraft navigation problems.

Thus, the developed method of iterative formation of selective reference images for CENS by the "sliding window" method, using the correlation analysis of images based on brightness, can be applied in CENS with external sensors, which form an image in the visible optical, infrared and radar range. The disadvantages of the method may include relatively high requirements for computational resources. The RI preparation time (depending on the power of the calculator) can take several hours. It is therefore appropriate to use this approach for the preparation of reference images in cases where there are no rigid time requirements for the task.

\subsection{Development of binary reference images}

An important requirement for RI is to minimize the amount of memory it occupies, which can be achieved by creating and using selective images.One way to reduce the amount of memory occupied by RI is to form binary RI.Formally, to obtain binary images (based on the images in the "grey wedge"), it is sufficient to define a certain brightness threshold - $L_{B}$. Next, for each pixel of the image in the "grey wedge", the following rule applies:

$$
S(i, j) b= \begin{cases}0, & \text { if } \quad S(i, j)<L_{B} \\ 1, & \text { if } \quad S(i, j) \geq{ }^{3} L_{B}\end{cases}
$$

where $S(i, j)$ is the brightness of the image at point $(i, j)$;
$S(i, j)^{b}$ is the brightness of the binary image at point $(i, j)$. However, in such a case the correlation between the neighbouring pixels of the image to be converted may be lost.In our case, selective images obtained by the method described above can be used to form binary RI.Then, for the selected images obtained, rule (3) is simplified:

$$
S(i, j) b=\left\{\begin{array}{ccc}
1, & \text { if } & S(i, j)>0 \\
0, & \text { in other cases }
\end{array}\right.
$$

where $S(i, j)$ is the brightness of the selective image at (i, j).

Figure 10, a) shows the binary RI derived from a selective image generated using a FCAB.Figure 10. b) shows the JCF of binary RI and OI.

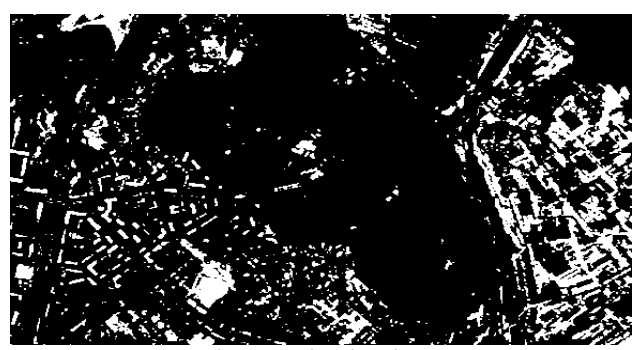

a) Binary selective image

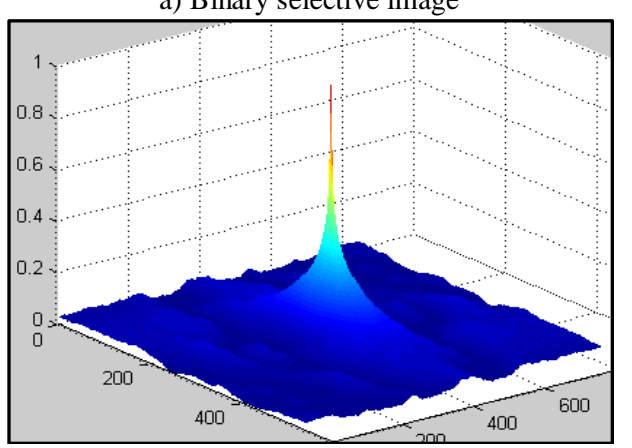

b) JCF of Original and binary images

Figure 10: Results of binary selective image production and calculation of the JCF of the given binary and original images.

As shown in the figure, the correlation between binary RI and $\mathrm{OI}$ is preserved.However, if the quality of the selective image based on the FCAB $L_{Q}=27$, then the binary RI is slightly lower - 23. The volume of said binary RI in the uncompressed form is $60 \mathrm{kB}$, and in the compressed form (with the aid of a data loss-free compression algorithm) is only $16 \mathrm{kB}$ (against a similar selective image, the volume of said binary RI in the compressed form is about $70 \mathrm{kB}$ )).

Note that the quality of the binary selective image (as with other RI obtained in this work) was assessed without any "useful" OI transformation.At the same time, certain "analytically simple" transformations may be carried out over the current image, which is formed by the external information sensors of the CENS, in order to better solve the problem of the flight correction of the aircraft.Such "simple" and hence rapid transformation may include threshold processing.

Figure 11 describes the OI in the "grey wedge" to which the threshold treatment was applied (threshold was 125 gray gradations (image had 256 such gradations), which is 
approximately 0,5 from the whole range of gradations) as well as the corresponding JCF for such OI and binary selective image. The $L_{Q}$ in this case reached a value of 39 , which is higher than the FCAB-based selective image with OI without previous processing.

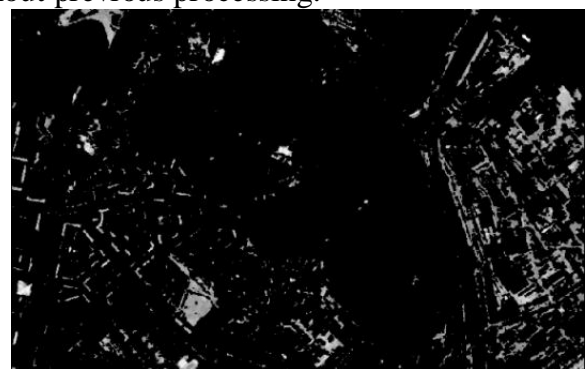

a) Binary selective image

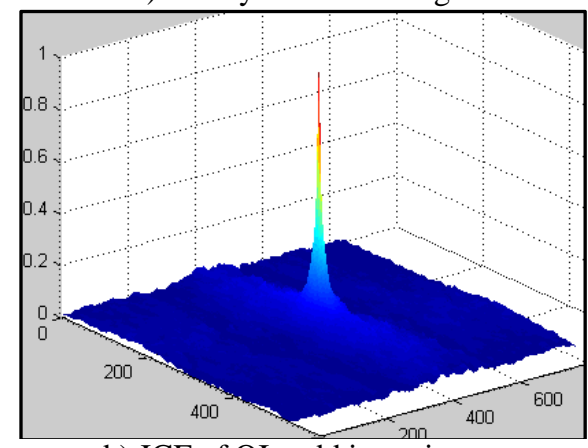

b) JCF of OI and binary images

Figure 11: Results of OI generation using threshold processing and calculation of the JCF of this original and binary images.

Since image binarization is also a "quick" operation, it applies to the image obtained after the threshold processing of the OI.The result of the transformation is shown in Figure 12.The $L_{Q}$ level in this case has reached a value of 40, which almost corresponds to the previous result. That is, binarization of OI (and therefore current images) is not a mandatory operation when using binary selective RI, unlike threshold processing, which significantly improves the result.

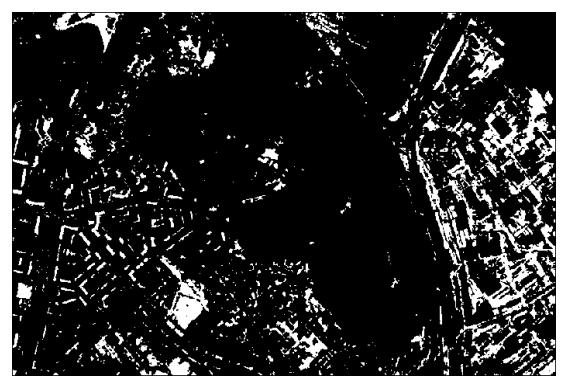

a) OI after threshold treatment

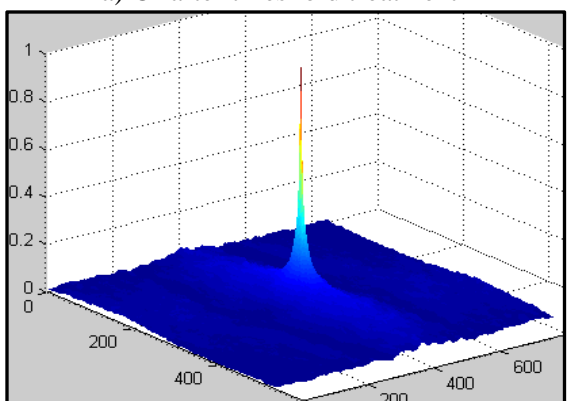

b) JCF ofbinary original and binary and selective images
Figure 12: Results of OI formation using threshold processing and binarization and calculation of the JCF of given original and binary selective images.

It should be noted that the procedure for selecting a threshold level for the on-board processing of current images for the use of binary RI remains an open question.It has been pointed out above that a selective image with fewer informative features produces JCF with a lower level of "background", a sharper main lobe and therefore a higher value of $L_{Q}$.We'll check that on other images.

Figure 13 shows different (FCAB-level) selective images and corresponding of JCF to Olin a "grey wedge".

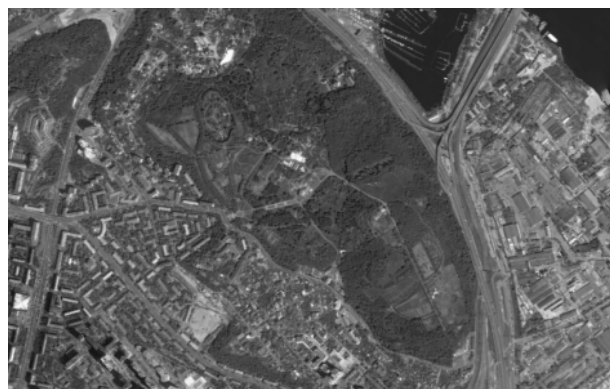

a) Original image

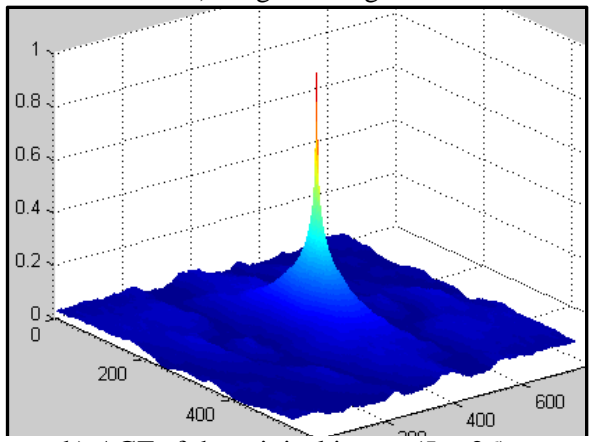

b) ACF of the original image $\left(L_{O}=26\right)$;

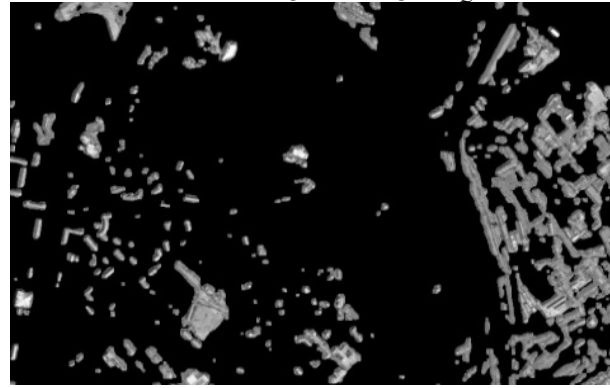

c) Selective image

(FCAB section at 0.5 )

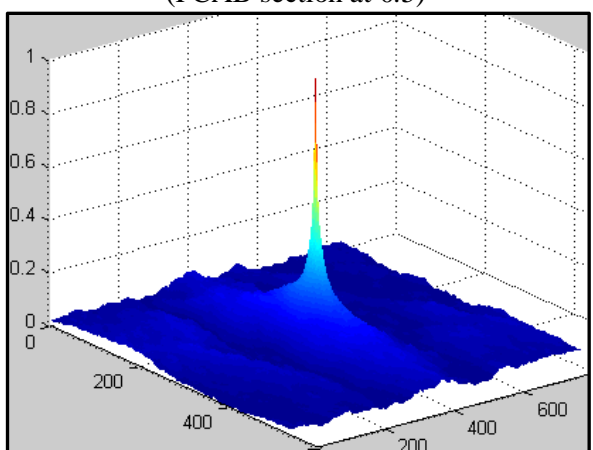

d) JCF of OI and selective image $\left(L_{Q}=27\right)$; 


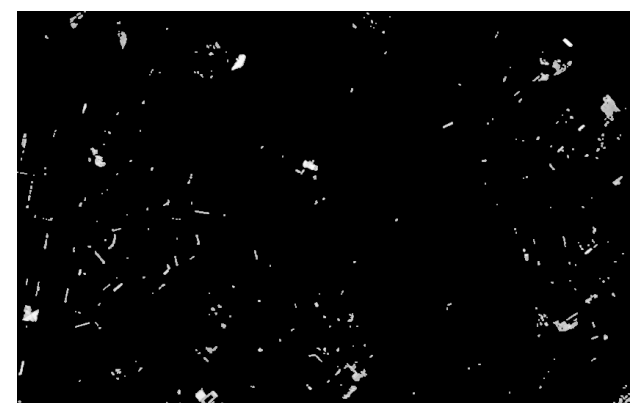

e) Binary selective image (FCAB section at 0.7);

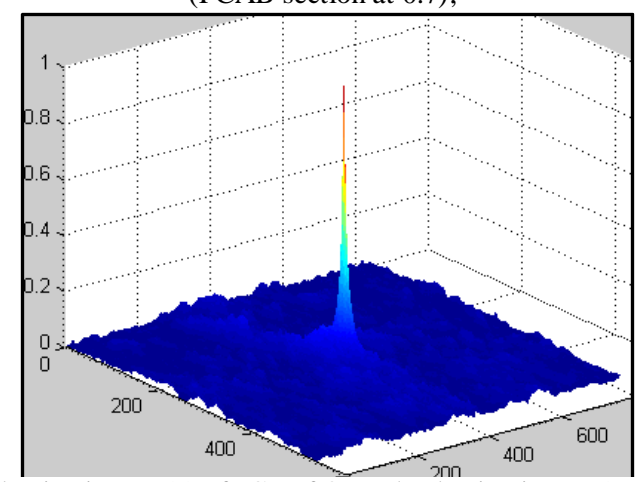

f) Selective image (e) of JCF of OI and selective image $\left(L_{Q}=28\right)$;

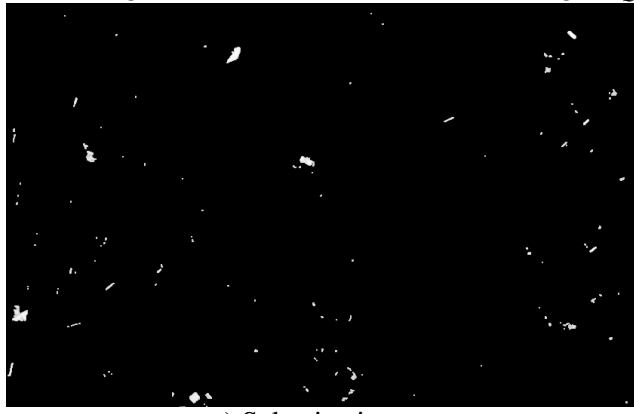

g) Selective image

(FCAB section at 0.8 )

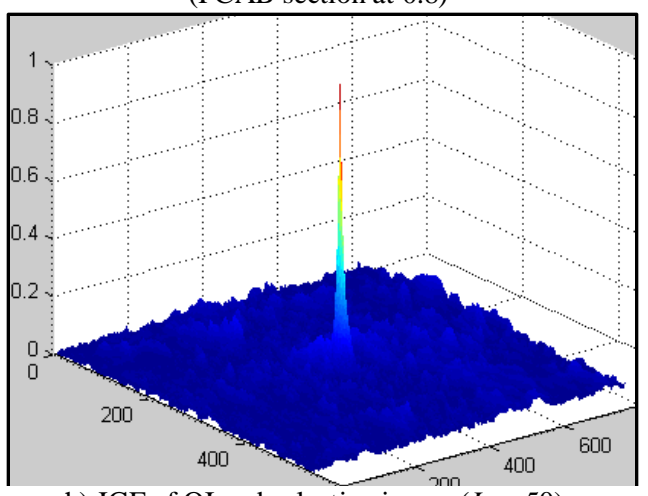

h) JCF of OI and selective image $\left(L_{Q}=50\right)$;

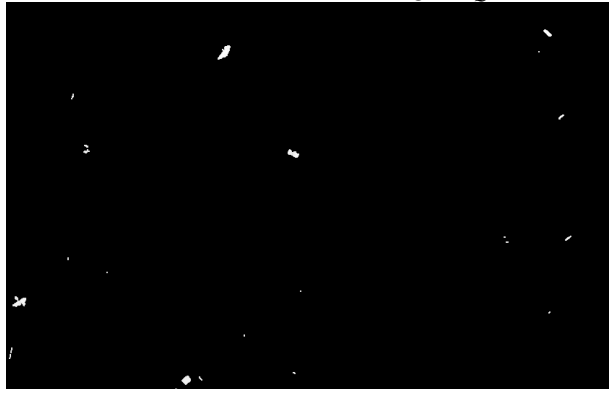

i) Selective image

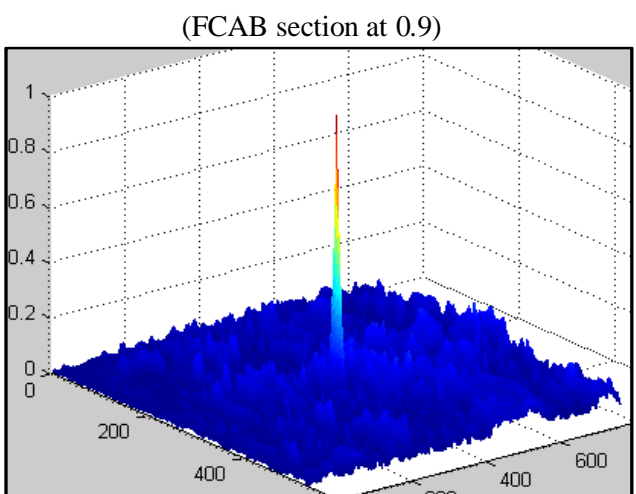

j) JCF of OI and selective image $\left(L_{Q}=32\right)$.

Figure 13: Selective images and their JCF with the original image in a «grey wedge».

Figure 13 shows that the "impoverishment" of a selective image on informative grounds improves the quality of the JCF.This is due to the faster decrease in the background level of the JCF (due to the "emptiness" in the selective image) relative to the fall in the level of its main lobe.After a certain limit, the level of the main lobe of the JCF falls more rapidly and its quality deteriorates. This confirms the need for an iterative search for the best RI of possible alternatives.

Figure 14 shows the result of calculating the JCF for the same selective images after converting to binary and selective source images. The results presented in the figure confirm the above conclusion.

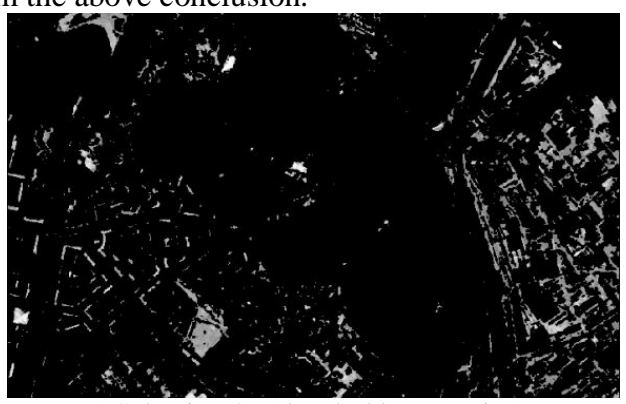

a) Selective OI (threshold processing)

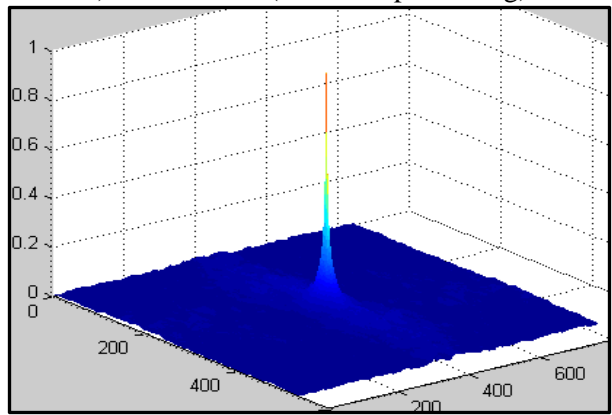

b) ACF of selective $\mathrm{OI}\left(L_{Q}=81\right)$

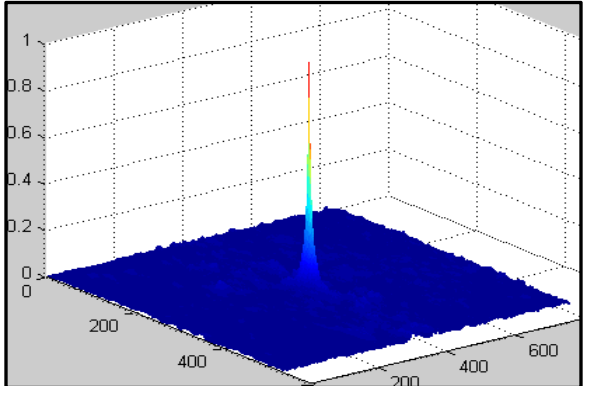


c) JCF of OI and selective image $\left(L_{Q}=103\right)$

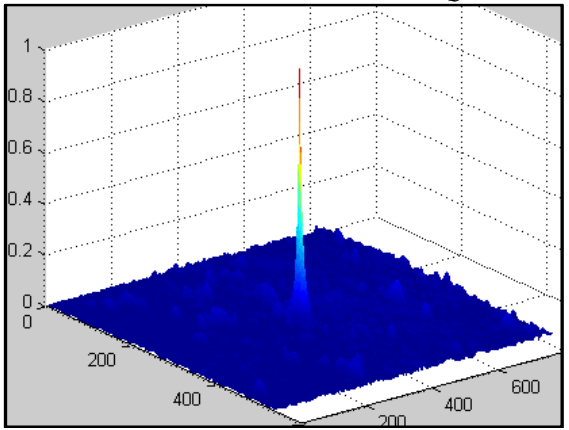

d) JCF of OI and selective image $\left(L_{Q}=89\right)$

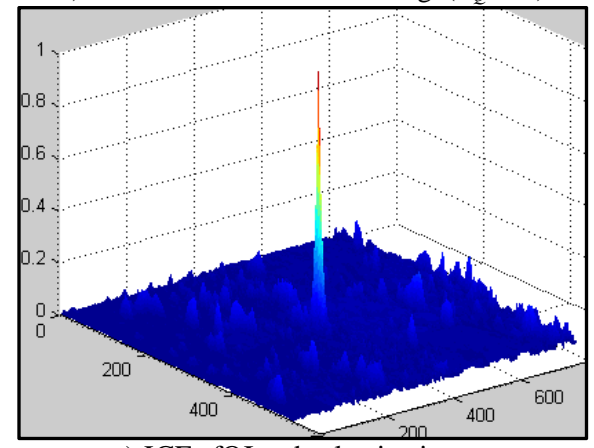

e) JCF ofOI and selective image $\left(L_{Q}=57\right)$.

Figure 14: Selective images and their JCF with selective original image in «grey wedge»

Figure 14 shows that the process of threshold processing for OI (as well as for RI consequently on board of the aircraft) allows to perform more accurate flight correction of the aircraft since the received JCF are indeed unimodal, their principal lobe is narrow (sharp-edged) andsubstantially dominates the "background". Moreover, the low level of side lobes of JCF reduces the possibility of incorrect binding of aircraft's CENS almost to zero.

\section{CONCLUSION}

As a result of the performed research the value of utilization of the "sliding window" method to form the RI in CENS is presented.

If there is a need to reduce the amount of computation, it is possible to use binary images with "sliding window" treatment, and it is shown that the rational choice of the cross-sectional level of the FCAB can ensure the required accuracy of the navigation of the aircraft.

\section{REFERENCES}

1. V. Antyufeev. Matrix radiometric correlationextreme navigation systems for aircraft: monographUkraine, Kharkov: KhNU V.N. Karazin, 372 p. 2. A. Sotnikov, V. Tarshyn, N. Yeromina, S. Petrov, N. Antonenko. A method for localizing a referenceobject in a current image with several bright objects, EasternEuropean Journal of Enterprise Technologies. 2017. Vol. № 9 (87). pp. 68-74. DOI: $10.15587 / 1729$ 4061.2017.101920.

3. N.Yeromina, S. Petrov, A. Tantsiura, M. Iasechko, V. Larin. Formation of reference images and decisionfunction in radiometric correlation-extremal navigation systems. Eastern-European Journal ofEnterprise Technologies. - 2018. Vol.4, No.9 (94). pp. 27-35. DOI: 10.15587/1729-4061.2018.139723.

4. O.Tymochko , A.Ttystan , V.Ushan , N.Yeromina , O.Dmitriiev, V.Mazharov, I.Padalka , I.Hannoshyna , I. Masik, A. Zazirnyi The Synthesis of the Reference Image and Algorithms for Vehicle Navigation Systems,JETER,8(3), 2020, pp. 853- 858. doi: 10.30534/ijeter/2020/40832020.

5. Y. Bryzhatyi, H. Kovalov, V.Dachkovskyi, L.MikhailovaThe Operation of Detection Systems in Conditions of Contrast Decrease of Ground Objects, JETER, 8 (1), 2020, pp. 208-212, doi: 10.30534/ijeter/2020/28812020

6. N.Yeromina, S. Petrov, A. Tarasenko, N. Antonenko, O. Semenenko, O. Pavlovskyi, M. Sliusarenko, V. TelehinThe Use of Strong and Ordinal Scales during the Synthesis of Reference Images for Vehicle Correlation-Extreme, IJATCSE, 9 (2), 2020, pp. 23432349, doi: 10.30534/ijatcse/2020/218922020

7. N. Yeromina, S. Petrov, N. Antonenko, I. Vlasov, V. Kostrytsia, V. KorshenkoThe Synthesis of the Optimal Reference Image Using Nominal and Hyperordinal Scales, JETER, 8 (5), 2020, pp. 2080-2084, doi: 10.30534/ijeter/2020/98852020

8. O. Liashko, V. Klindukhova, N.Yeromina, T.Karadobrii, O.Bairamova, A. DoroshevaThe Criterion and Evaluation of Effectiveness of Image Comparison in Correlation-Extreme Navigation Systems of Mobile Robots, JETER, 8 (6), 2020, pp. 2841-2847, doi: 10.30534/ijeter/2020/97862020

9. Smelyakov, K., Hvozdiev, M., Chupryna, A., Sandrkin, D., Martovytskyi, V.Comparative Efficiency Analysis of Gradational Correction Models of Highly Lighted Image //2019 IEEE International ScientificPractical Conference Problems of Infocommunications, Science and Technology (PIC S \& amp;T). - IEEE, 2019. - C. 703-708. 